\title{
Umbilical cord blood stem cells as a beneficial option in cell-based therapy and regenerative medicine
}

\begin{abstract}
Stem cells are unique cells obtained from a broad range of tissues with different features related to proliferation capability and differentiation capacity. As one of the most important stem cell types in medicine today, umbilical cord blood-derived stem cells (UCB-SCs) are a more practical choice than embryonic stem cells because their use does not involve ethical issues. For example, these cells are a powerful tool used in cell-based therapy and regenerative medicine to treat cancer and neurological, autoimmune, cardiovascular, and blood diseases. In this article, we perform a mini review of umbilical cord blood-derived stem cells as an effector option in cell-based therapy and regenerative medicine.
\end{abstract}

Keywords: umbilical cord blood-derived stem cells, umbilical cord blood-derived mscs, umbilical cord blood-derived hscs, regenerative medicine, cell-based therapy

\author{
Volume 3 Issue 2 - 2017
}

\begin{abstract}
Ali Kowsari, Zahra Jafarian, Reza Tabatabaei Qomi, Azar Sheikholeslami, Naser Kalhor, Hoda Fazaeli, Mohsen Sheykhhasan

Department of Stem Cell,The Academic Center for Education, Iran
\end{abstract}

Correspondence: Mohsen Sheykhhasan, Researcher at Department of Stem Cell, The Academic Center for Education, Culture and Research, Qom, Iran,

Email m.sheykhhasan@acecr.ac.ir

Received: June 29, 2017 | Published: July 25, 2017
Abbreviations: UCB-SCs, umbilical cord blood-derived stem cells; HSCs, hematopoietic stem cells; MSCs, mesenchymal stem cells; UCB-MSCs, umbilical cord blood-derived mesenchymal stem cells; UCB-MSCs, umbilical cord blood-derived mscs; hUCB-MSCs, human umbilical cord blood-derived mscs; GvHD, graft-versus-host disease; HIV, human immunodeficiency virus

\section{Introduction}

\section{Umbilical cord blood}

Umbilical cord blood used to be regarded as waste material after childbirth. ${ }^{1}$ In the last decades, however, it has been found to contain valuable biomaterials such as stem cells. ${ }^{2-5}$ These potentially lifesaving cells in umbilical cord blood are called hematopoietic stem cells (HSCs). ${ }^{6,7}$ This blood contains mesenchymal stem cells (MSCs) as well ${ }^{8-10}$ (Figure 1).

\section{Umbilical cord blood-stem cells benefits and features}

Both of the abovementioned stem cells derived from umbilical cord blood could be used to repair and restore damaged tissues and treat diseases such as cancer, blood and neurological disorders, and immune deficiencies because of their tremendous proliferation and differentiation potential and great regenerative capacity. ${ }^{11-26}$ In addition, these candidate cells have a great paracrine mechanism on other cells. ${ }^{27}$ This biological mechanism could increase stem cells' regenerative capacity. ${ }^{27}$

Besides these biological mechanisms, umbilical cord blood-derived MSCs (UCB-MSCs) have few immunological properties because they naturally express little class I MHC and lack HLA-DR and both CD80 and CD86 proteins. ${ }^{28-32}$ A number of studies have shown that UCBMSCs have initial immunomodulatory effect and are compatible with safety systems..$^{28-32}$ These mechanisms are unknown, but evidence shows that paracrine factors secreted by these candidate cells could be involved in this process. ${ }^{28-30}$ UCB-MSCs immunomodulatory effects on the immune system provide by the number of paracrine factors. ${ }^{27-30}$ These factors not only inhibit T-cell proliferation but also often increase anti-inflammatory cytokine secretions. ${ }^{28-30}$ Owing to this regulatory effect, UCB-MSCs regulate immune responses with anti-inflammatory effects and expand production. ${ }^{30}$ Consequently, the use of these candidate cells in the allogeneic form could be suggested as an immunologically safe treatment in the field of regenerative medicine ${ }^{30}$ (Figure 2).

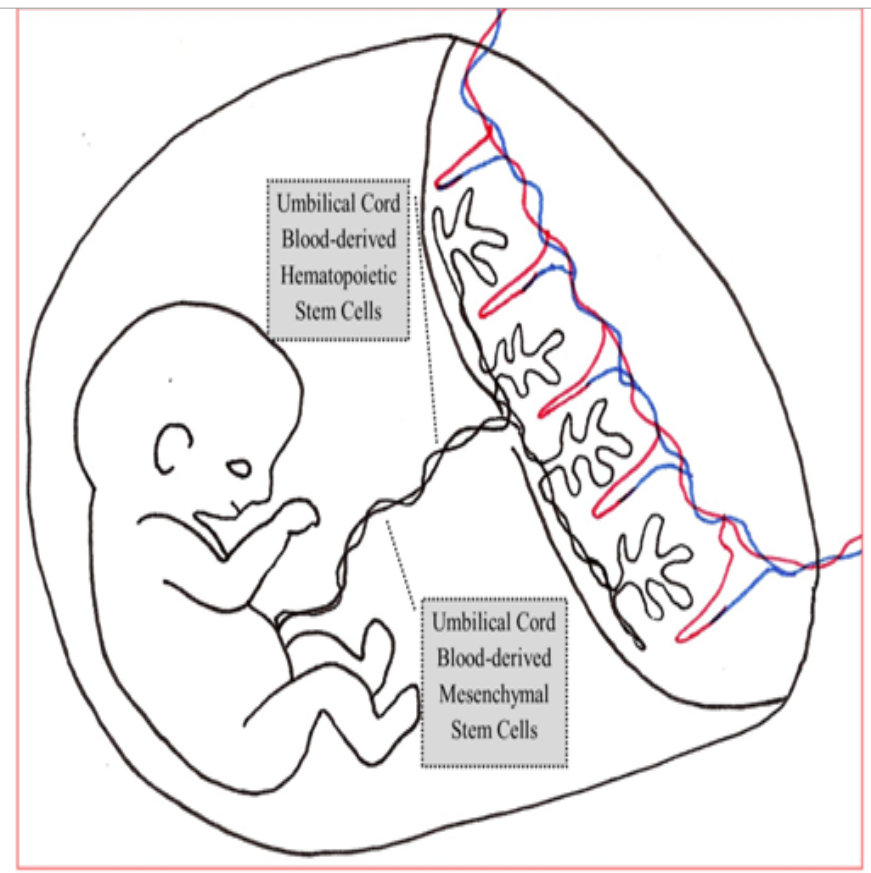

Figure I This figure schematically demonstrates the different types of stem cells from umbilical cord blood. 


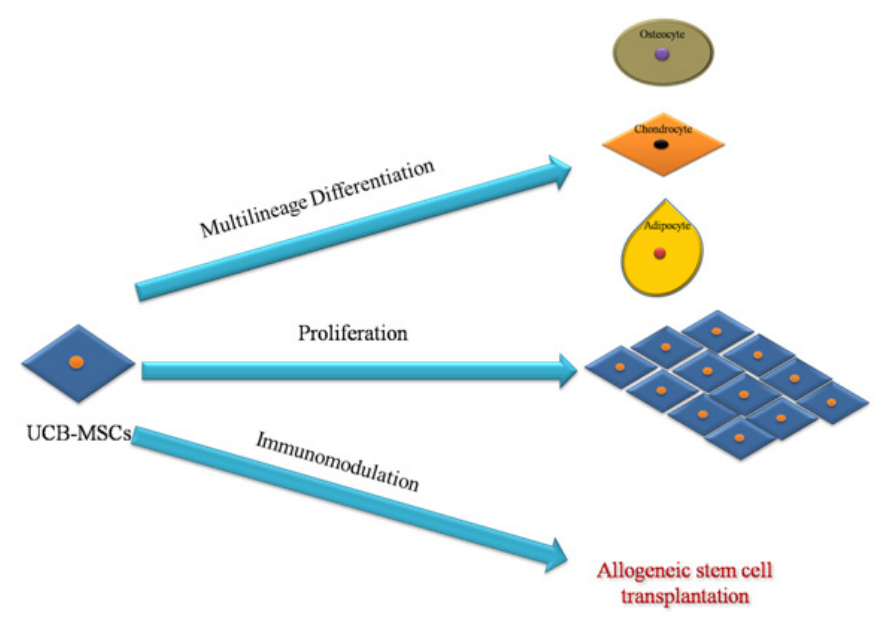

Figure $\mathbf{2}$ This figure schematically demonstrates the unique properties of umbilical cord blood-derived mesenchymal stem cells for regenerative medicine approach.

Similar to MSCs derived from bone marrow, UCB-MSCs can differentiate into multilineage cells (e.g., adipocyte, chondrocyte and osteocyte) at induced differentiation conditions in vitro (Figure 2) ${ }^{28}$ In addition to UCB-MSCs, hematopoietic stem cells are other stem cells derived from umbilical cord blood that can generate red blood cells, white blood cells, and platelets ${ }^{33}$ (Figure 3). Furthermore, UCB-HSC could be differentiated into hematological cells and non-hematological cells in physiologic and pathological condition, respectively. ${ }^{34}$

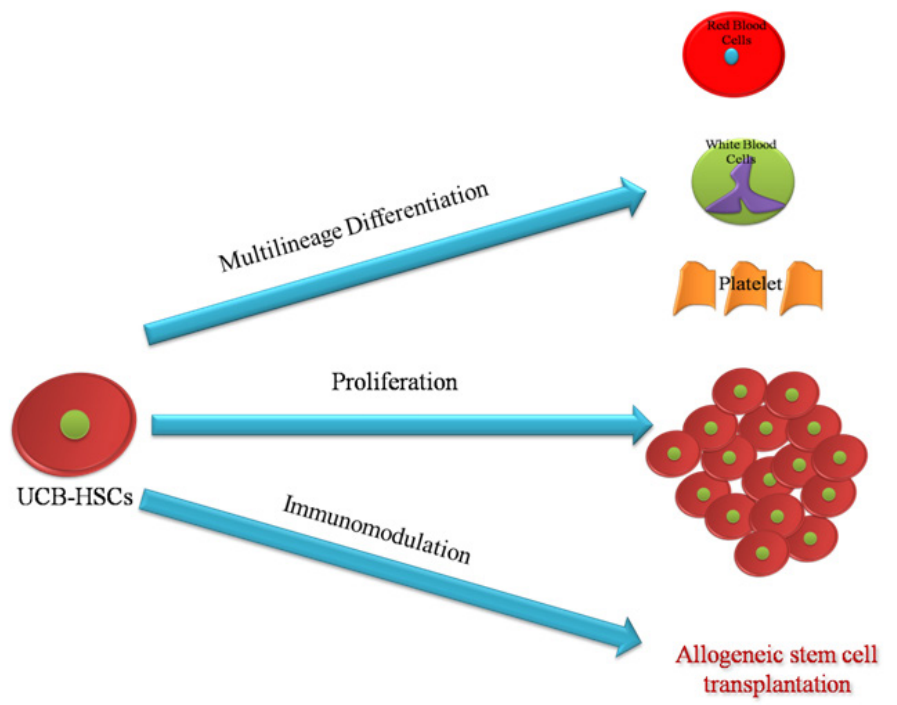

Figure 3 This figure schematically demonstrates the unique properties of umbilical cord blood-derived hematopoietic stem cells for regenerative medicine approach.

UCB-MSCs holds the ability of genes expression associated with embryonic stem cells, and, through this feature, they differ from adult stem cells. ${ }^{35}$ Additionally, these cells show more proliferation than adult stem cells. ${ }^{35}$ As a result, these cells prefer the culture condition related to embryonic stem cells as compared to adult stem cells. ${ }^{35}$

Due to UCB-HSC' great therapeutic capacity in the field of cellbased therapy, many private/commercial and public cord blood banks have been established worldwide. ${ }^{34}$ UCB-HSC are collected, processed, and cryo-reserved in cord blood banks. ${ }^{34}$ For the use of cryo-reserved UCB-HSC in cord blood banks, it is important to assess several factors. ${ }^{34}$ HLA matching is one of the most important factors measured for UCB-HSC transplantation. ${ }^{34}$ In addition to HLA matching, the dose or content of some cells, including CD34+ cells or colony-forming cells, are required to determine the hematopoietic recovery after UCB stem cell transplantation. ${ }^{34}$ Furthermore, the total nucleated cell count are of critical importance for UCB stem cell transplantation. ${ }^{34}$

\section{Applications of umbilical cord blood-stem cells in re- search study}

An animal-based study showed that the human umbilical cord's mesenchymal stem cells may be useful in treatments for alleviating the symptoms of preeclampsia. ${ }^{36}$ In this study, human umbilical cord cells were collected, extracted, and cultured. ${ }^{36}$ The results of these experiments demonstrated that umbilical cord blood's mesenchymal stem cells have the ability to affect preeclampsia symptoms in the mice. ${ }^{36}$ Additionally, the blood pressure reduced and fetal weight symptoms associated with the disease increased after treatment. ${ }^{36}$ Finally, the outcomes of this study demonstrated that the transfer of UCB-MSC may be useful for the treatment of preeclampsia. ${ }^{36}$

Treatment using UCB-MSCs has shown promise for treating diabetes mellitus in a rat model, particularly in regard to the vitreous humor and the blood-retinal barrier. ${ }^{37}$ Furthermore, it has been demonstrated that MSCs derived from human umbilical cord blood (hUCB-MSCs) may have a positive effect on murine experimental colitis. ${ }^{38}$

Three experiments carried out on two dimensional conditions have illustrated that UCB-HSC could have better maintained under specific condition, such as hypoxic condition. ${ }^{39-41}$ However, some studies described that $3 \mathrm{D}$ culture systems could be used in proliferation and differentiation of UCB stem cells for tissue engineering applications. ${ }^{42-44}$

Therefore, during various experiments, HSC stem cells were proliferated and expanded on a variety of three-dimensional (3D) conditions, including electrospun polyethersulfone nanofibers, 3D collagen gels, PCL, PLGA, fibrin, collagen, hydroxyapatite ceramic scaffolds, macroporous PEG hydrogels and so on. ${ }^{42-45}$

In an in vitro study, chondrogenic differentiation ability of human UCB-MSCs was evaluated in four different scaffolds during 4 weeks. ${ }^{46}$ Considering the obtained outcome in this study, it was identified that human fibroblast-derived matrix (hFDM) had the best effect on the cell differentiation and mesenchymal condensation in vitro. ${ }^{46}$

An in vitro experiment also confirmed that the UCB-MSCs could be induced to differentiate toward osteoblast lineage on $2 \mathrm{D}$ and $3 \mathrm{D}$ environment. ${ }^{47}$ In this study, the osteogenic differentiation of UCBMSCs was accelerated on both 2D and 3D environment by single and dual growth factors delivery using nanoparticles-in-microcapsules. ${ }^{47}$

\section{Umbilical cord blood-stem cell in the clinical study}

Most of the clinical trials related to UCB-MSCs are in phases I and II, in which the safety and efficacy of treatment is determined. ${ }^{48}$ The efficacy of treatment for all studies based on UCB-MSCs has shown no serious adverse events or side effects. ${ }^{1}$

Recently, stem cells derived from umbilical cord blood have been 
clinically transplanted into patients with severe thalassemia and some types of cancer, including malignant and non-malignant disorders. ${ }^{49-51}$ Thus far, it appears that, worldwide, more than 600,000 transplants have been performed using stem cells obtained from umbilical cord blood. ${ }^{7}$ It is important to mention that most completed or ongoing clinical trials are still in the early phases (phase I and II).$^{48}$ As a result, the outcomes of these studies are not yet clear.

According to data obtained from ClinicalTrials.gov, more than 180 clinical trials have demonstrated the widespread use of umbilical cord blood stem cells for the treatment of diseases. ${ }^{48}$ These clinical studies are verified based on the beneficial results of the most recent studies. One of the most important clinical trials focuses on neurological disorders such as autism, cerebral palsy, spinal cord damage, hearing loss and hypoxic ischemic encephalopathy, as well as autoimmune medical conditions including multiple sclerosis, lupus, diabetes mellitus, Crohn's disease, graft-versus-host disease (GvHD), and rheumatoid arthritis (Figure 4) ${ }^{51}$ Cardiovascular diseases such as congenital heart disease, ischemic stroke, and myocardial infarction have also been treated using these candidate cells (Figure 4) ${ }^{51}$ Additionally, inherited disorders such as human immunodeficiency virus (HIV), thalassemia, sickle cell disease, and severe combined immunodeficiency can be treated by UCB-MSCs. ${ }^{51}$ In addition to these diseases, UCB-MSCs also were used for treat orthopedic problems, including articular cartilage defects and knee articular cartilage injury. ${ }^{51}$

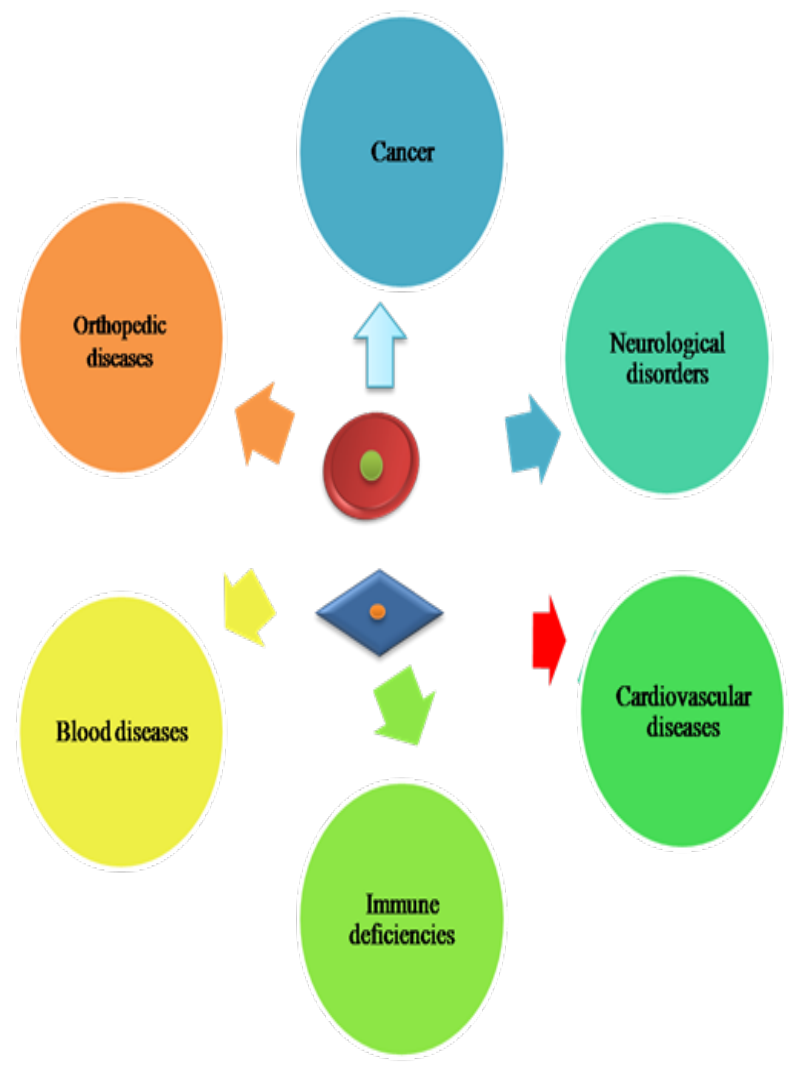

Figure 4 This figure schematically demonstrates the application of umbilical cord blood stem cells for regenerative medicine approach.

\section{Conclusion}

The future holds great promise for the use of umbilical cord blood stem cells. In the transplantation discipline, collection of data from clinical trials will continue to improve knowledge, thereby assisting to enhance survival rates. Recent medical progress have illustrated that these candidate cells could be used to treat the similar disease as the hematopoietic stem cells available in bone marrow but without some of the drawbacks of these type of transplant. These candidate cells are currently used to treat approximately 80 diseases including cancer, immune deficiencies, cardiovascular diseases, neurological disorders, and blood diseases.

\section{Acknowledgements}

None.

\section{Conflict of interest}

The author declares no conflict of interest.

\section{References}

1. Malgieri A, Kantzari E, Patrizi MP, et al. Bone marrow and umbilical cord blood human mesenchymal stem cells: state of the art. Int J Clin Exp Med. 2010;3(4):248-269.

2. Waller-Wise R. Umbilical cord blood: information for childbirth educators. J Perinat Educ. 2011;20(1):54-60.

3. Armson BA. Umbilical cord blood banking: implications for perinatal care providers. J Obstet Gynaecol Can. 2005;27(3):263-290.

4. Bordet S, Nguyen TM, Knoppers BM, et al. Use of umbilical cord blood for stem cell research. J Obstet Gynaecol Can. 2010;32(1):58-61.

5. Bojanić I, Golubić Cepulić B. Umbilical cord blood as a source of stem cells. Acta Med Croatica. 2006;60(3):215-225.

6. McKenna D, Sheth J. Umbilical cord blood: current status \& promise for the future. Indian J Med Res. 2011;134:261-269.

7. Ballen KK, Verter F, Kurtzberg J. Umbilical cord blood donation: public or private? Bone Marrow Transplant. 2015;50(10):1271-1278.

8. Gong X, Wang P, Wu Q, et al. Human umbilical cord blood derived mesenchymal stem cells improve cardiac function in cTnT(R141W) transgenic mouse of dilated cardiomyopathy. Eur J Cell Biol. 2016;95(1):5767.

9. Matsumoto T, Mugishima H. Non-hematopoietic stem cells in umbilical cord blood. Int J Stem Cells. 2009;2(2):83-89.

10. Zarrabi M, Mousavi SH, Abroun S, et al. Potential uses for cord blood mesenchymal stem cells. Cell J. 2014;15(4):274-281.

11. Achyut BR, Varma NR, Arbab AS. Application of Umbilical Cord Blood Derived Stem Cells in Diseases of the Nervous System. J Stem Cell Res Ther. 2014;4:1000202.

12. Park SK, Won JH. Usefulness of umbilical cord blood cells in era of hematopoiesis research. Int J Stem Cells. 2009;2(2):90-96.

13. Iafolla MA, Tay J, Allan DS. Transplantation of umbilical cord bloodderived cells for novel indications in regenerative therapy or immune modulation: a scoping review of clinical studies. Biol Blood Marrow Transplant. 2014;20(1):20-25.

14. Bari S, Seah KK, Poon Z, et al. Expansion and homing of umbilical cord blood hematopoietic stem and progenitor cells for clinical transplantation. Biol Blood Marrow Transplant. 2015;21(6):1008-1019.

15. Damien P, Allan DS. Regenerative Therapy and Immune Modulation Using Umbilical Cord Blood-Derived Cells. Biol Blood Marrow Transplant. 2015;21(9):1545-1554. 
16. Wagner JE, Brunstein CG, Boitano AE, et al. Phase I/II Trial of StemRegenin-1 Expanded Umbilical Cord Blood Hematopoietic Stem Cells Supports Testing as a Stand-Alone Graft. Cell Stem Cell. 2016;18(1):144 155.

17. Fu L, Liu Y, Zhang D, et al. Beneficial effect of human umbilical cordderived mesenchymal stem cells on an endotoxin-induced rat model of preeclampsia. Exp Ther Med. 2015;10(5):1851-1856.

18. Wang LL, Yu Y, Guan HB, et al. Effect of Human Umbilical Cord Mesenchymal Stem Cell Transplantation in a Rat Model of Preeclampsia. Reprod Sci. 2016;23(8):1058-1070.

19. Meng HB, Gong J, Zhou B, et al. Therapeutic effect of human umbilical cord-derived mesenchymal stem cells in rat severe acute pancreatitis. Int J Clin Exp Pathol. 2013;6(12):2703-2712.

20. Roura S, Pujal JM, Gálvez-Montón C, et al. The role and potential of umbilical cord blood in an era of new therapies: a review. Stem Cell Res Ther. 2015;6:123.

21. El Omar R, Beroud J, Stoltz JF, et al. Umbilical cord mesenchymal stem cells: the new gold standard for mesenchymal stem cell-based therapies? Tissue Eng Part B Rev. 2014;20(5):523-544.

22. Ruggeri A, Ciceri F, Gluckman E, et al. Eurocord and Acute Leukemia Working Party of the European Blood and Marrow Transplant Group. Alternative donors hematopoietic stem cells transplantation for adults with acute myeloid leukemia: Umbilical cord blood or haploidentical donors? Best Pract Res Clin Haematol. 2010;23(2):207-216.

23. Ballen KK, Gluckman E, Broxmeyer HE. Umbilical cord blood transplantation: the first 25 years and beyond. Blood. 2013;122(4):491-498

24. Screnci M, Murgi E, Valle V, et al. Sibling cord blood donor program for hematopoietic cell transplantation: the 20-year experience in the Rome Cord Blood Bank. Blood Cells Mol Dis. 2016;57:71-73.

25. Hordyjewska A, Popiołek $Ł$, Horecka A. Characteristics of hematopoietic stem cells of umbilical cord blood. Cytotechnology. 2015;67(3):387396.

26. Wu J, Sun Y, Block TJ, et al. Umbilical cord blood-derived non-hematopoietic stem cells retrieved and expanded on bone marrow-derived extracellular matrix display pluripotent characteristics. Stem Cell Res Ther. 2016;7(1):176.

27. Pineault N, Abu-Khader A. Advances in umbilical cord blood stem cell expansion and clinical translation. Exp Hematol. 2015;43(7):498-513.

28. Nagamura-Inoue T, He H. Umbilical cord-derived mesenchymal stem cells: Their advantages and potential clinical utility. World J Stem Cells. 2014;6(2):195-202.

29. Friedman R, Betancur M, Boissel L, et al. Umbilical cord mesenchyma stem cells: adjuvants for human cell transplantation. Biol Blood Marrow Transplant. 2007;13:1477-1486.

30. Deuse T, Stubbendorff M, Tang-Quan K, et al. Immunogenicity and immunomodulatory properties of umbilical cord lining mesenchymal stem cells. Cell Transplant. 2011;20(5):655-667.

31. Prasanna SJ, Gopalakrishnan D, Shankar SR, et al. Pro-inflammatory cytokines, IFNgamma and TNFalpha, influence immune properties of human bone marrow and Wharton jelly mesenchymal stem cells differentially. PLoS One. 2010;5:e9016.

32. Lee M, Jeong SY, Ha J, et al. Low immunogenicity of allogeneic human umbilical cord blood-derived mesenchymal stem cells in vitro and in vivo. Biochem Biophys Res Commun. 2014;446(4):983-989.

33. van de Ven C, Collins D, Bradley MB, et al. The potential of umbilical cord blood multipotent stem cells for nonhematopoietic tissue and cell regeneration. Exp Hematol. 2007;35(12):1753-1765.
34. Ilic D, Miere C, Lazic E. Umbilical cord blood stem cells: clinical trials in non-hematological disorders. Br Med Bull. 2012;102:43-57.

35. Kanji S, Pompili VJ, Das H. Plasticity and maintenance of hematopoietic stem cells during development. Recent Pat Biotechnol. 2011;5(1):40-53.

36. Wang LL, Yu Y, Guan HB, et al. Effect of Human Umbilical Cord Mesenchymal Stem Cell Transplantation in a Rat Model of Preeclampsia. Reprod Sci. 2016;23(8):1058-1070.

37. Dong M, Zhang W, Chen S, et al. The protective effect of human umbilical cord mesenchymal stem cells-induced neural stem cells in the vitreous on the blood-retinal barrier in diabetic rats. Zhonghua Yan Ke Za Zhi. 2017;1:53-58.

38. Kim HS, Yun JW, Shin TH, et al. Human umbilical cord blood mesenchymal stem cell-derived PGE2 and TGF- $\beta 1$ alleviate atopic dermatitis by reducing mast cell degranulation. Stem Cells. 2015;33(4):1254-1266.

39. Roy S, Tripathy M, Mathur N, et al. Hypoxia improves expansion potential of human cord blood-derived hematopoietic stem cells and marrow repopulation efficiency. Eur J Haematol. 2012;88(5):396-405.

40. Danet GH, Pan Y, Luongo JL, et al. Expansion of human SCID-repopulating cells under hypoxic conditions. J Clin Invest. 2003;112(1):126135

41. Ivanovic Z, DelloSbarba P, Trimoreau F, et al. Primitive human HPCs are better maintained and expanded in vitro at 1 percent oxygen than at 20 percent. Transfusion. 2000;40(12):1482-1488.

42. Wendt D, Marsano A, Jakob M, et al. Oscillating perfusion of cell suspensions through three-dimensional scaffolds enhances cell seeding efficiency and uniformity. Biotechnol Bioeng. 2003;84(2):205-214.

43. Raic A, Rödling L, Kalbacher H, et al. Biomimetic macroporous PEG hydrogels as 3D scaffolds for the multiplication of human hematopoietic stem and progenitor cells. Biomaterials. 2014;35(3):929-940.

44. Ferreira MS, Jahnen-Dechent W, Labude N, et al. Cord blood-hematopoietic stem cell expansion in 3D fibrin scaffolds with stromal support. Biomaterials. 2012;33(29):6987-6997.

45. Gluckman E, Ruggeri A, Volt F, et al. Milestones in umbilical cord blood transplantation. Br J Haematol. 2011;154:441-447.

46. Kim IG, Ko J, Lee HR, et al. Mesenchymal cells condensation-inducible mesh scaffolds for cartilage tissue engineering. Biomaterials. 2016;85:18-29.

47. Subbiah R, Hwang MP, Van SY, et al. Osteogenic/angiogenic dual growth factor delivery microcapsules for regeneration of vascularized bone tissue. Adv Healthc Mater. 2015;4(13):1982-1992.

48. Amir Ali Hamidieh, Tahereh Rostami, Mohammadreza Ostadali, et al Outcomes of Unrelated Donor Hematopoietic Stem Cell Transplantation for Pediatric Patients:The First Report From Iran. Biology of Blood and Marrow Transplantation. 2017;23(3):S122.

49. Amir Ali Hamidieh, Maryam Behfar, Amir Eslami, et al. TBI-Free Pediatric Hematopoietic Stem Cell Transplantation in Iran: Two Decades of Practice. Biology of Blood and Marrow Transplantation. 2016;22(3):S321-S322.

50. Ghavamzadeh A, Alimoghaddam K, Jahani M, et al. Hematopoietic Stem Cell Transplantation For Major Thalassemia: Nineteen Years Experience In Iran. Biology of Blood and Marrow Transplantation. 2010;16(2):S159.

51. El-Badri N. Advances in stem cell therapy: bench to bedside. USA: Humana Press; 2017. 